\title{
CALCULATING INTERNAL AVALANCHE VELOCITIES FROM CORRELATION WITH ERROR ANALYSIS
}

\author{
J. N. MCELWAINE \\ Department of Applied Mathematics and Theoretical Physics, Centre for Mathematical Sciences, \\ Wilberforce Road, Cambridge, CB3 OWA, UK \\ E-mail: jnm11@amtp.cam.ac.uk \\ F. TIEFENBACHER \\ Swiss Federal Institute for Snow and Avalanche Research, Flüelastrasse 11, CH-7260, Davos Dorf, \\ Switzerland \\ E-mail: tiefenbacher@slf.ch
}

(Received 20 March 2003; Accepted 14 April 2003)

\begin{abstract}
Velocities inside avalanches have been calculated for many years by calculating the crosscorrelation between opto-electronic sensors using a method pioneered by Nishimura et al. (1987) and Dent et al. (1998). Their approach has been widely adopted but there has been little discussion of the optimal design of such instruments and the best analysis techniques. This paper discusses some of the different sources of error that arise and how these can be mitigated. A statistical framework that describes such instruments is developed and used to quantify the errors.
\end{abstract}

Keywords: correlation methods, granular flows, opto-electronic sensors, snow avalanches, velocity profiles

\section{Introduction}

Opto-electronic sensors have been used for a long time to measure the velocities inside granular flows. Some of the earliest work was done by Nishimura et al. (1987) on snow avalanches and continued in Nishimura et al. (1993). Early work was also done by Dent et al. (1998) and measurements were taken from the "Revolving Door" avalanche path near Bridger Bowl, Montana.

The basic design of these sensors is simple. An infrared LED emits light that is reflected by the passing granular material and this is detected by an infraredsensitive photo-transistor, amplified, digitised and stored on a computer. By comparing the signals from nearby sensors it is possible to calculate the velocity of the flow.

In theory it is possible to calculate many other pieces of information about the flow since the magnitude of the back scattered light depends on the density, type, size, and orientation of the snow crystals. However, though Dent et al. (1998) tried to relate reflectivity to snow density, they failed because crystal size and type are much more important than density. Some gross aspects of the flow can be determined, however. For example in deposited snow the signal will be constant, in a 

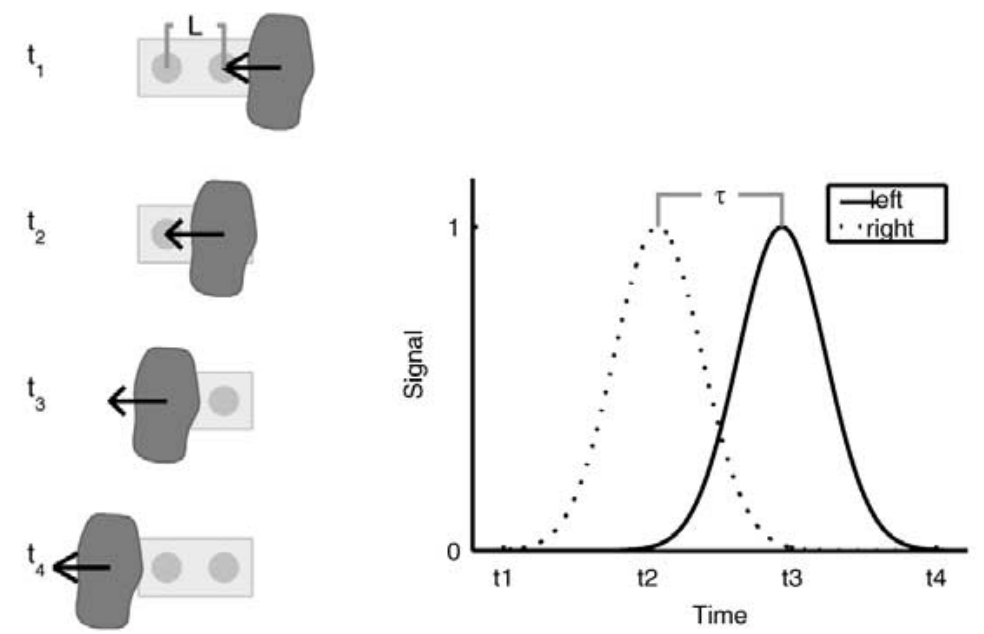

Figure 1. Schematic of a typical instrument and measurement process for a single particle. Estimated velocity is $L / \tau$.

powder cloud the signal will be very low since no light from above can reach the sensor and the density is usually too low to reflect light significantly, and above the snow a high level will be detected due to ambient lighting.

Despite the wide spread use of opto-electronic sensors there appears to have been little work done on analysing the errors and designing instruments so as to minimise them. In this paper we describe how to calculate the errors for these sensors. We then use these results to discuss how these sensors should best be designed. Some of the different variables considered are the number of sensors, sensor geometry, relative position, size and sample rate. Before we can calculate the errors it is necessary to first discuss how the data are to be processed; for this to be done a model for the behaviour of the flow field is necessary.

For convenience in this paper we ignore discretization errors in both time and space and regard all functions as continuous. Only when considering the numerical implementation will a discrete time approach be used. This simplification can be made as long as the signals are properly filtered before digitisation so that there are no frequencies higher than the Nyquist frequency (half the sample frequency).

We start by discussing the most frequently used method, the cross-correlation method, and explain why this method can be unreliable. Then we introduce a modified method and discuss the errors for a simple two point sensor. This shows that there are several main sources of errors. The most serious is due to the twodimensional nature of real flow fields past a flat sensor, whereas the analysis assumes one-dimensional flow. This error can be reduced by careful designing of the sensors or explicitly accounting for two-dimensional velocities. The second source of error is due to changes of the velocity. The correlation method assumes that the velocity is constant over the time window used for calculating the cross-correlation; 


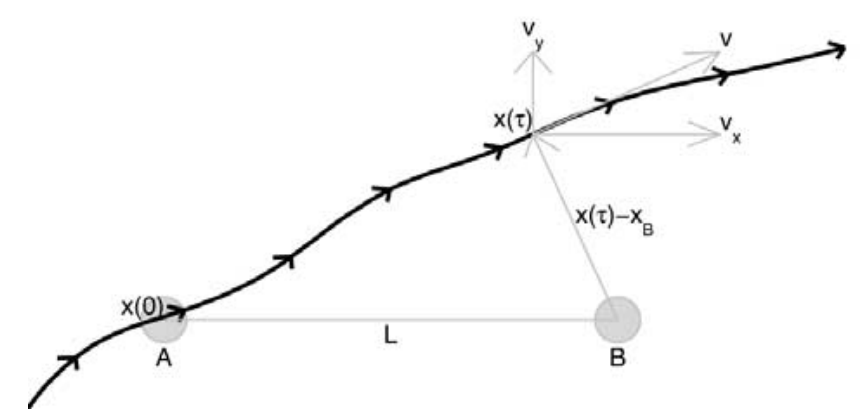

Figure 2. Particle trajectory with maximum correlation occurring at point of closest approach

when this is not true, because the flow is accelerating, errors are introduced. The acceleration errors can be reduced by improved analysis techniques where the velocity is calculated as a changing function at all times. Note that the acceleration can be caused by convective changes in velocity, that is different parts of the flow are moving with different velocities, or temporal acceleration, that is the velocity field is changing with time. Thirdly, there are errors due to the inherent statistical velocity fluctuations in a granular flow. The mean velocity in a granular medium is only defined once an averaging process over individual particles has been specified. Measurements of the mean velocity based on averaging over a region of space or time will have statistical uncertainty. There are also errors introduced by the aperture effect, which will be explained later in greater depth. Internal changes in the flowing snow will also cause inaccurate correlations. And in a similar vein velocities normal to the mounting plane of the sensor will also introduce errors in the correlation. Since sensors are normally mounted on large walls and the normal velocity must vanish at the surface this effect will be very small unless the sensor is sensitive to a few particle diameters into the flow.

\section{Errors in Interpreting the Lag}

We assume that the largest cross-correlation between two sensors will occur at a time when a region of the flow which passed over the first sensor is at its closest to the second. This is discussed in more detail in McElwaine (2003) and shown to be true in the mean. This region of the flow does not have to be a particular particle, but only a region with an identifiable reflectivity signature, but for simplicity we will talk about a particle. Figure 2 shows the trajectory of this particle that is over sensor $A$ at time 0 so that $\mathbf{x}(0)=\mathbf{x}_{A}$ and is closest to sensor $B$ at time $\tau$. Disregarding internal changes in the material and false matches the correlation between sensors $A$ and $B$ will be greatest at lag $\tau$. The point of closest approach of the trajectory 


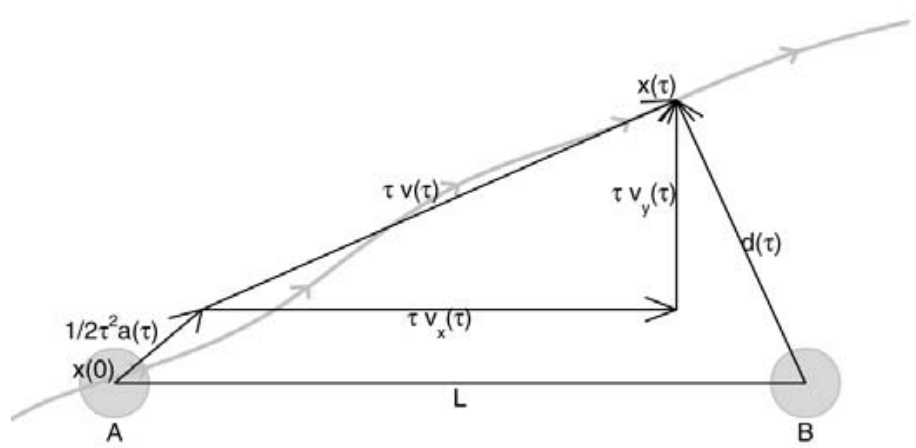

Figure 3. The dependence of $\tau$ on the particle trajectory can also be calculated geometrically. In the case when acceleration, a, can be ignored then two applications of Pythagoras' theorem give $\tau^{2} v^{2}+d^{2}=L^{2}$ and $\tau^{2} v_{y}^{2}+\left(L-\tau v_{x}\right)^{2}=d^{2}$. Eliminating $d^{2}$ between the two equations gives $L / \tau=v_{x}+v_{y}^{2} / v_{x}$.

to sensor $B$ occurs when the displacement between them is perpendicular to the tangent of the trajectory, which is of course the velocity. Thus

$$
\left[\mathbf{x}(\tau)-\mathbf{x}_{B}\right] \cdot \mathbf{v}(\tau)=0 .
$$

The same equation results algebraically by differentiating to find the minimum of the distance $\left|\mathbf{x}(\tau)-\mathbf{x}_{B}\right|$. We then define the effective particle acceleration $\mathbf{a}$ by

$$
\mathbf{x}(t)=\mathbf{x}_{A}+t \mathbf{v}(\tau)+t^{2} \mathbf{a}(t) / 2 .
$$

That is $\mathbf{a}(\mathbf{t}) t^{2} / 2$ is the difference in position between the actual position at time $t$ and the position if the particle had moved with constant velocity $\mathbf{v}(\tau)$ (see Figure 3.) Taking the dot product of Equation 2 with $\mathbf{v}(\tau)$ and evaluating at $t=\tau$ this becomes

$$
\mathbf{x} \cdot \mathbf{v}-\mathbf{x}_{A} \cdot \mathbf{v}=v^{2} \tau+\tau^{2} \mathbf{a} \cdot \mathbf{v} / 2,
$$

where $\mathbf{x}$ and $\mathbf{a}$ are evaluated at $t=\tau$. Then using Equation 1 to eliminate $\mathbf{x} \cdot \mathbf{v}$ and we have

$$
\left(\mathbf{x}_{B}-\mathbf{x}_{A}\right) \cdot \mathbf{v}=v^{2} \tau+\tau^{2} \mathbf{a} \cdot \mathbf{v} / 2 .
$$

If we take the sensors to be separated by a distance $L$ along the $\mathrm{x}$ axis then this becomes $L v_{x}=\tau v_{x}^{2}+\tau v_{y}^{2}+\tau^{2}\left(a_{x} v_{x}+a_{y} v_{y}\right) / 2$ which can be rearranged to give

$$
v_{x}=\frac{L}{\tau}-v_{y} \frac{v_{y}+\tau a_{y} / 2}{v_{x}}-\tau a_{x} / 2
$$

Thus, if the particle is travelling with constant velocity $\mathbf{v}$ along the $x$-axis (the sensors' axis) so that $a_{y}, v_{y}$ and $v_{x}$ are all zero, then $v_{x}=L / \tau$, which is the usual 


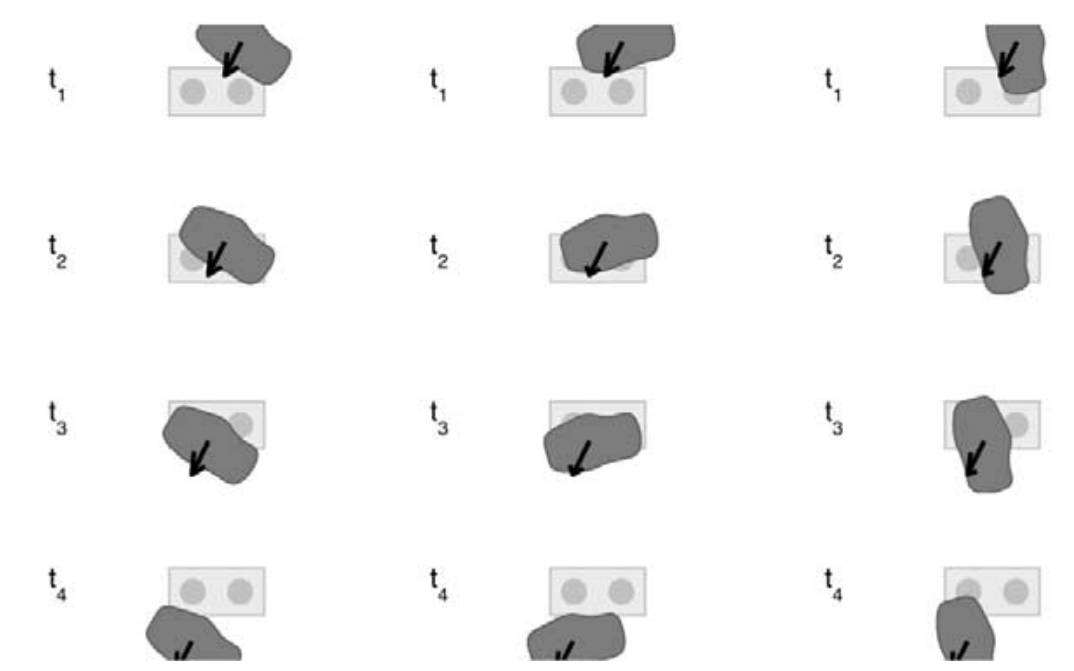

Figure 4. Different orientations of a particle with the same velocity give rise to different correlation times. In (a) the particle is moving orthogonally to its edge and the correlation time will be positive. In (b) the particle is rotated anti-clockwise and the correlation time will be negative. In (c) the particle is rotated clockwise giving a larger positive correlation time.

formula. In other cases, however, the velocity estimate $\hat{v}_{x}=L / \tau$ will be in error by $v_{y}\left(v_{y}+\tau a_{y} / 2\right) / v_{x}-\tau a_{x} / 2$. For low accelerations the main contribution will be from the velocity component $v_{y}$ which biases the estimate high by $v_{y}^{2} / v_{x}$.

Equation 5 concerns point velocities at time $\tau$ and has a displacement error term a which is similar to the averaged particle acceleration. Another approach is to attempt to calculate the particle's average velocity over the time interval $\tau$ so we define

$$
\overline{\mathbf{v}}=\frac{\mathbf{x}(\tau)-\mathbf{x}(0)}{\tau} .
$$

But now the point velocity $\mathbf{v}(\tau)$ is not known and this must be approximated by defining a new pseudo-acceleration $\mathbf{a}^{\prime}$ by

$$
\mathbf{v}(\tau)=\overline{\mathbf{v}}+\tau \mathbf{a}^{\prime} .
$$

Substituting this relation into Equation 1 and rearranging gives

$$
\bar{v}_{x}=\frac{L}{\tau}-\bar{v}_{y} \frac{\bar{v}_{y}+\tau a_{y}^{\prime}}{\bar{v}_{x}}+a_{x}^{\prime}\left(\tau-\frac{L}{\bar{v}_{x}}\right) .
$$

This is very similar to Equation 5. The significant difference is that the estimate of velocity is accurate to first order in $a_{x}^{\prime}$ since $a_{x}^{\prime}\left(\tau-\left(L / \bar{v}_{x}\right)\right)=O\left(a_{x}^{\prime 2} L^{2} / \bar{v}_{x}^{3}\right)$. This is not surprising; it shows that the output from these instruments will best be 


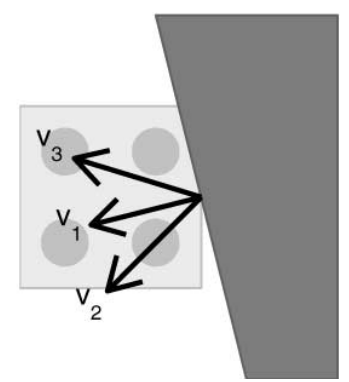

Figure 5. All three velocities $v_{1}, v_{2}$ and $v_{3}$ will result in the same lags between the sensors. No sensor array can distinguish between them and only the velocity component perpendicular to the edge can be measured.

regarded approximations to velocity averaged over the lag time rather than point velocities.

\section{Errors in Calculating the Lag}

The analysis in the previous section assumed that the lag with the maximum crosscorrelation occurred when the same part of the flow was identified as it passed near each sensor. This is only true on average and is not the case in general. There are two main effects to be considered, which are discussed in the following subsections.

\subsection{APERTURE EFFECT}

Each photo-transistor measures the reflected intensity of the material, thus regions in the flow with the same reflectivity cannot be distinguished. Rather than seeing the correlation between different points in the flow the sensors in fact measure the correlation between contours of equal reflectivity. These contours of equal reflectivity correspond to the edges of particles. Therefore only the velocity component orthogonal to the reflectivity contours can be measured (see Figure 5). This difficulty is known as the aperture effect Jähne (1997) (Chapter 13).

In the case of only two sensors the problem is even worse since the normal direction $\hat{\mathbf{n}}$ cannot be calculated. A simple calculation shows this effect explicitly (see Figure 6) so that

$$
\hat{v}_{x}=\frac{L}{\tau}=\frac{v \cos (\theta-\phi)}{\cos \theta}=\frac{\mathbf{v} \cdot \hat{\mathbf{n}}}{\hat{\mathbf{x}} \cdot \hat{\mathbf{n}}},=v_{x}+v_{y} \tan \theta,
$$

where $\hat{\mathbf{x}}$ is the unit vector along the $x$-axis. This shows that vertical velocities can increase or decrease the estimate $\hat{v}_{x}$ depending on the angle of the edge. This effect is shown in Figure 4 where the same particle approaches the sensor with same velocity but different orientations. 


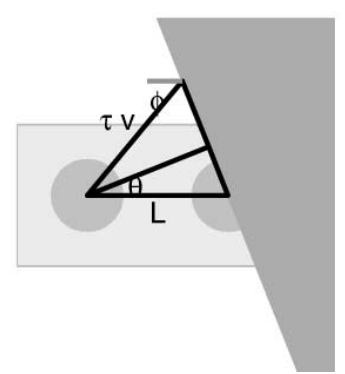

Figure 6. Diagram showing how the lag time $\tau$ depends on the direction of the velocity $\phi$, the speed $v$ and the direction of the edge normal $\theta . L \cos \theta=\tau v \cos (\theta-\phi)$.

This subsection has discussed the relationship between the lag $\tau$, the velocity of the flow, and its structure when the lag is calculated from individual events. Different approaches for calculating the lag will be discussed later, but these all calculate the lag by comparing the signal over large time intervals which therefore average over the different orientations and velocities in the flow and thus act to mitigate these effects.

\subsection{Spatial Aliasing}

If there are spatial variations less than the spacing between the sensors, then the Nyquist limit is violated and aliasing can occur. The surface area of the sensors acts as an averaging, low-pass filter so it is not the distance between the centres of the sensors that must be small but the distance between the edges of the active part of the sensors. The requirement can be simply stated that there should be no room for particles to pass between the sensors without giving a signal. The most obvious effect of this will be when there are significant vertical velocities, and then there may be no correlation between the sensors at all. This is shown in Figure 7. In this case the critical length between which there are no correlations $L_{c}$ is the sum of the particle diameter and sensor diameter. This problem is an example of spatial aliasing. If there are wavelengths in the signal smaller than the sampling wavelength errors occur. To combat this problem the diameter of the sensors must be increased, which corresponds to low-pass filtering, or the sensors must be moved closer together, which corresponds to decreasing the sampling wavelength. If the particle diameter is $L_{c}$, the separation of the sensors $L$ and the diameter of the sensors $L_{s}$, the Nyquist criterion for non-aliased sampling is roughly $L_{s}+L_{c} \geq L$. If it is known that the vertical velocities will be much less than horizontal velocities this criterion can be relaxed to $L_{s}+L_{c} \geq L\left|v_{x}\right| /\left|v_{y}\right|$. This condition of small vertical velocities will be discussed in the next section. 
$t 1$

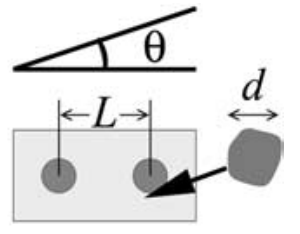

$t 2$

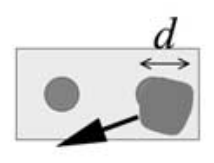

t3

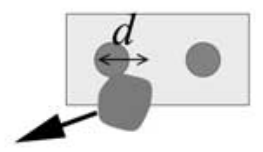

Figure 7. Vertical motion can give rise to spatial aliasing. There will only be correlations between the sensor elements if $L \sin \theta<d$.

\section{Analysis Methods}

\subsection{CROSS-CORRELATION}

This is the approach that has been used by most authors including Dent et al. (1998) and Tiefenbacher and Kern (2003). The cross-correlation of two sequences $\left\{x_{i}\right\}$ and $\left\{y_{i}\right\}$ at lag $j$ is defined as

$$
\rho(j)=\frac{\sum_{i \in A}\left(x_{i}-\bar{x}\right)\left(y_{i+j}-\bar{y}_{j}\right)}{\sqrt{\sum_{i \in A}\left(x_{i}-\bar{x}\right)^{2} \sum_{i \in A}\left(y_{i+j}-\bar{y}_{j}\right)^{2}}} .
$$

The sums are taken over a time interval $A$ of width $n$ samples, for example $k-$ $n / 2 \ldots k+n / 2 . \bar{x}$ and $\bar{y}$ are the window averages. Sometimes it is not always clear exactly how these have been defined but for consistency this should be $\bar{x}=$ $1 / n \sum_{i} x_{i}$ and $\bar{y}_{j}=1 / n \sum_{i} y_{i+j}$. Other variants that are symmetrical between $x$ and $y$ are possible. The velocity is then calculated by finding the value of $j$ that maximises $\rho(j)$, and then the velocity is estimated using $\hat{v}_{x}=L v_{s} / j$, where $v_{s}$ is the sampling frequency and $L$ the distance between the sensors. The velocity is usually thought of as being the velocity calculated at time $k v_{s}$, but a more accurate approximation would be to take it as the velocity at time $v_{s}(k+j / 2)$. In either case this suggests that the velocity can only be calculated as lying in intervals of width $L v_{s}$ and that this is a fundamental design restriction. This is not the case since $\rho(j)$ can be approximated at any value of $j$ using interpolation to gain additional precision.

A strength of this method is that it is invariant to shifts and scaling of the signal, and it is straightforward. That is, the same lag will be found for $a x+b$ 
and $c y+d$ for all non-zero constants $a, b, c$ and $d$. This is useful if each combined sensor and amplifier system has different gains and offsets, since this method will automatically account for this. However, the duration of any experiment is usually no more than a few minutes and over this period the electronics should be stable and show little drift. This means that the offset and gain can be chosen once for the whole sequence and should be accurate. Even if this is not possible, it would be better first to perform a filtering and transformation step, to normalise the data, and then to calculate the correlation. This will only fail if one of the sensors changes in some way as the avalanches passes, for example if some snow sticks to the front of one sensor, but not the other. This effect can easily be investigated by comparing the signal level before and after the avalanche.

Subtracting the mean from each signal before calculating the cross-correlation is similar to applying a high-pass filter. It throws away an important part of the signal: its absolute value. It is easy to construct examples where this method will perform badly. This is shown in Figure 8. The signals are identical triangular waves with a lag of $0.1 \mathrm{~s}$ with a very small amount (standard deviation $1 \times 10^{-3}$ ) of zero mean Gaussian noise. A window width of $0.3 \mathrm{~s}$ was used, corresponding to 1000 samples. Smaller window widths give even worse results. The search range was restricted to $\pm 1.5 \mathrm{~s}$. Larger search ranges give larger errors. The errors can be eliminated by choosing a window width that is greater than the period $(1 \mathrm{~s})$. What this figure shows is that, provided the window includes a turning point from each signal, the correct lag is calculated, and the error increases smoothly with the noise. In this example the noise is so small that the error is not visible. The correlation function calculated for the time a is shown in the lower left figure and shows a well defined peak at the correct lag. The middle lower figure shows the correlation function at time b. In this case the two peaks are only just contained at the edge of the window and the maximum is much less distinct. The lower right figure shows the correlation function calculated at time c. Now the two peaks are not in the window. Where neither peak is in the window the two functions match almost perfectly so the correlation function is almost 1 . For large lags there is one turning point included and the correlation falls off. The reason for this behaviour is obvious. By subtraction of the mean and normalising by the mean squared deviation any two straight lines are brought into a perfect match. This means that this method is only sensitive to changes in the curvature (second derivative) of the signal.

\subsection{Modelling THE SignAl}

To perform any kind of analysis, the first step is understanding what we measure. Since sensors are generally fixed on approximately flat plates which are impermeable to the flow the local velocity normal to the sensor will be zero. So that to a good approximation the flows can be treated as two dimensional. Since little is known about the reflectivity of snow or other granular materials, we make no assumptions about the reflectivity field. Instead we assume a general function $f(\mathbf{x}, t)$, 

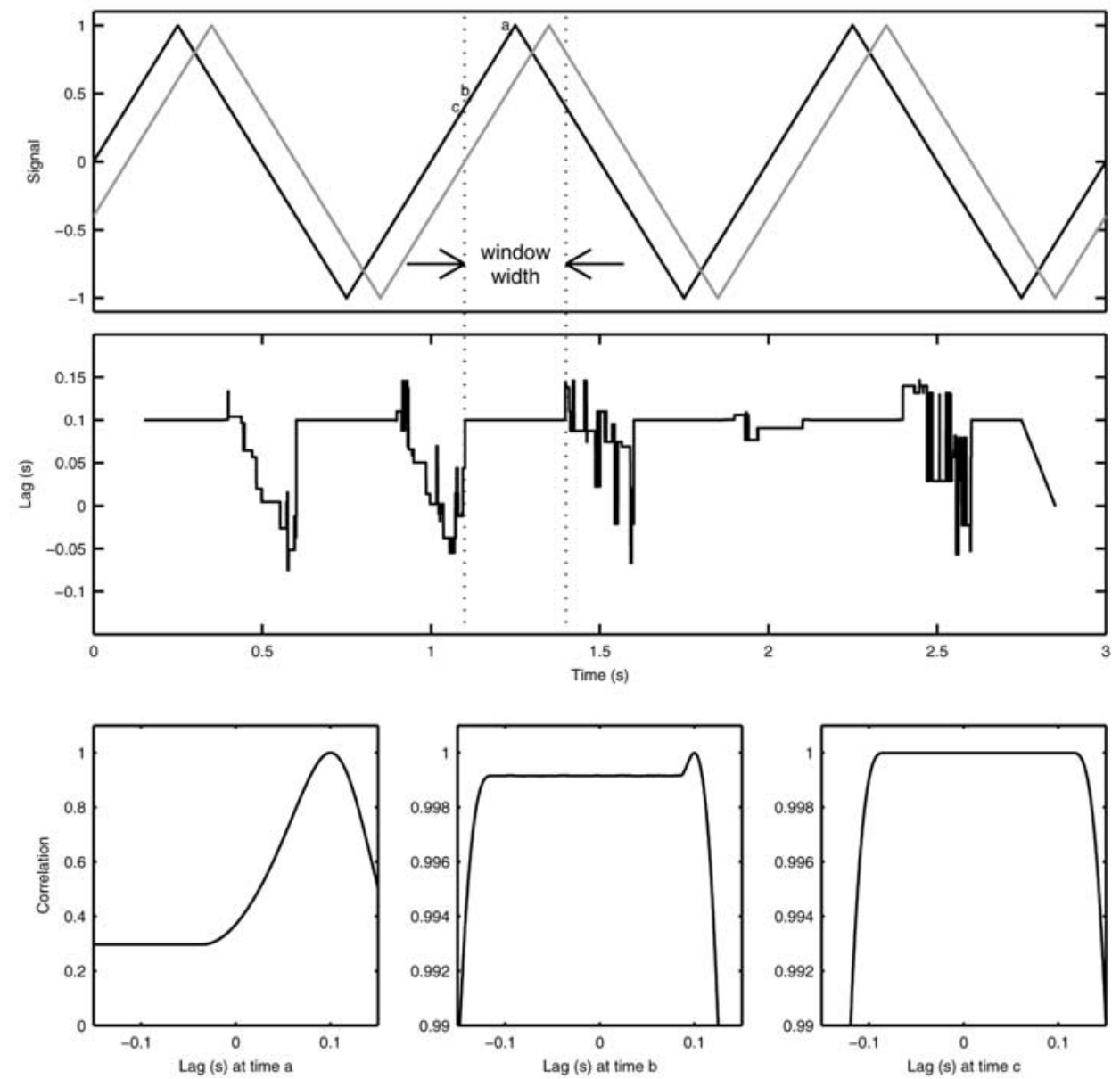

Figure 8. Failure of cross-correlation approach. The upper graph shows two nearly identical signals with a $0.1 \mathrm{~s}$ lag. The middle graph shows the calculated lag using maximum cross-correlation. The lower three figures show the cross-correlation function at the three points $\mathrm{a}, \mathrm{b}$ and $\mathrm{c}$ marked in the upper figure.

which depends on space and time, that specifies the signal that would be output from the sensor if it were positioned in the flow at position $\mathbf{x}=(x, y)$ at time $t$. This value will be a combination of the reflectivity of the snow smoothed over some volume in space according to the sensor's size, the amplification, the temporal smoothing, the digitising and finally the normalisation. This signal is then advected according to the flow velocity while changing only slowly due to three-dimensional effects and physical processes between the grains. $f$ satisfies the advection equation

$$
\frac{\partial f}{\partial t}+\mathbf{v} \cdot \nabla f \approx 0
$$


Equation 11 is the starting point for any analysis. In this paper we will not consider spatial variations in the velocity field $\mathbf{v}(\mathbf{x}, t)$. These can be included but considerably complicate the analysis without introducing any new features. Given this assumption, Equation 11 is easily solved. The characteristics of this equation are the trajectories of particles and since the velocity does not depend on position there is only one trajectory

$$
\mathbf{x}_{t}(t)=\int_{0}^{t} \mathbf{v}(s) d s .
$$

And then

$$
f\left(\mathbf{x}+\mathbf{x}_{t}(t), t\right)=f(\mathbf{x}, 0),
$$

or

$$
f(\mathbf{x}, t)=f\left(\mathbf{x}-\mathbf{x}_{t}(t), 0\right) .
$$

This means that a sensor at position $\mathbf{x}_{\mathbf{i}}$ will output a signal

$$
f_{i}(t)=f\left(\mathbf{x}_{i}, t\right)=f\left(\mathbf{x}_{i}-\mathbf{x}(t), 0\right)=f\left(\mathbf{x}_{i}-\mathbf{x}(t)\right),
$$

where $f$ is evaluated at $t=0$ when no time argument is given.

Since the $f_{i}$ are normalised to have mean zero, we can assume that the mean of $f$ is zero and define the auto-correlation function as

$$
\begin{aligned}
c(\mathbf{x}) & =E[f(\mathbf{x}+\mathbf{y}, t) f(\mathbf{y}, t)] \\
& =\frac{1}{A} \iint_{A} f(\mathbf{x}+\mathbf{y}, t) f(\mathbf{y}, t) \mathbf{d} \mathbf{y} \\
& =\frac{1}{T} \int_{0}^{T} f(\mathbf{x}+\mathbf{y}, t) f(\mathbf{y}, t) d t .
\end{aligned}
$$

The auto-correlation function can be defined as an average over different fields $f$ realised in different experiments, or as an average over space at one point in time, or as an average over time for two fixed points. If we assume ergodicity, stationarity and homogeneity these will be equivalent. These conditions will not be exactly true, but we can hope that they will be approximately true and check the accuracy later. The success of the method does not rely on them holding, but they are useful simplifying assumptions for the analysis. We also assume isotropy so that $c(\mathbf{x})$ is a function only of $|x|$. These rather technical assumptions considerably simplify the analysis and are natural. If more data become available they can be tested. Different degrees of variation in downslope and slope-normal directions can easily be incorporated by scaling the coordinates. 
Since a drawback of the cross-correlation method in Section 4.1 was that it was invariant under independent transformations of the functions, we consider a new method that is only invariant when both signals are transformed identically. This means that variations in mean levels and gradients will give a signal. We work in the continuous domain for convenience but it is straightforward to convert to the discrete domain using Fourier series.

\subsection{MINIMUM SQUARED DISCREPANCY}

A general approach to constructing analysis methods would attempt to have some of the properties of maximum likelihood estimation, that is minimising residuals. In this paper we will only consider two element sensors, but the method can be generalised. Then for two sensors separated by a distance $L$ we would expect $f_{1}(t+$ $\tau / 2)-f_{2}(t-\tau / 2)$ to be small when $\tau=L / v_{x}$. Thus we consider the method that minimises

$$
e(\tau)=\int\left[f_{1}(t+\tau / 2)-f_{2}(t-\tau / 2)\right]^{2} w(t) d t,
$$

where $w(t)$ is a weight function. Note that this is symmetric with respect to $f_{1}$ and $f_{2}$. If the integrand were unsymmetric with only one of the functions lagged, for example $f_{1}(t)-f_{2}(t-\tau)$, it can be shown that there are extra errors introduced that can be significant. The weight function $w(t)$ determines not only the width of the region over which the data are used but also its relative importance.

We will consider only gradual variations in the velocity, so we write

$$
\mathbf{x}_{t}(t)=\mathbf{v} t+\mathbf{a} t^{2} / 2,
$$

where $\mathbf{v}$ is the velocity at $t=0$ and $\mathbf{a}$ the acceleration. Using Equation 15

$$
f_{i}(t)=f\left(\mathbf{x}_{i}-\mathbf{v} t-\mathbf{a} t^{2} / 2\right) .
$$

Combining this with Equation 16 gives

$$
E\left[\left\{f_{1}(t+\tau / 2)-f_{2}(t-\tau / 2)\right\}^{2}\right]=2 c(0)-2 c(d(t, \tau)),
$$

where

$$
\begin{aligned}
d(t, \tau)= & \mid \mathbf{x}_{1}-\mathbf{x}_{2}-\mathbf{v}(t+\tau / 2)+\mathbf{v}(t-\tau / 2)-\mathbf{a}(t+\tau / 2)^{2} / 2 \\
& +\mathbf{a}(t-\tau / 2)^{2} / 2 \mid \\
= & \left|\mathbf{x}_{1}-\mathbf{x}_{2}-\mathbf{v} \tau-\mathbf{a} t \tau\right| .
\end{aligned}
$$

Therefore using Equation 16 


$$
\begin{aligned}
E[e(\tau)] & =\int E\left[\left\{f_{1}(t+\tau / 2)-f_{2}(t-\tau / 2)\right\}^{2}\right] w(t) d t \\
& =\int[c(0)-c(d(t, \tau))] w(t) d t .
\end{aligned}
$$

It is clear that this will be approximately minimised by choosing $\tau$ so as to minimise $d(t, \tau)$, thus justifying the earlier assumption that the maximum cross-correlation occurs at the point of closest approach.

In order to calculate the errors and the probability of failure (see Appendix A) we need to calculate $E[\dot{e}(\tau)]$ and $E[\ddot{e}(\tau)]$. Differentiating Equation 21 with respect to $\tau$ we have

$$
\begin{aligned}
\frac{d}{d \tau} d(t, \tau) & =\frac{d}{d \tau}\left|\mathbf{x}_{1}-\mathbf{x}_{2}-\mathbf{v} \tau-\mathbf{a} t \tau\right| \\
& =-\frac{(\mathbf{v}+t \mathbf{a}) \cdot\left(\mathbf{x}_{1}-\mathbf{x}_{2}-\mathbf{v} \tau-\mathbf{a} t \tau\right)}{d(t, \tau)},
\end{aligned}
$$

and

$$
\frac{d^{2}}{d \tau^{2}} d(t, \tau)=\frac{|\mathbf{v}+t \mathbf{a}|^{2}}{d(t, \tau)}+\frac{\left[(\mathbf{v}+t \mathbf{a}) \cdot\left(\mathbf{x}_{1}-\mathbf{x}_{2}-\mathbf{v} \tau-\mathbf{a} t \tau\right)\right]^{2}}{d(t, \tau)^{3}} .
$$

Thus

$$
E[\dot{e}(\tau)]=2 \int(\mathbf{v}+t \mathbf{a}) \cdot\left(\mathbf{x}_{\mathbf{1}}-\mathbf{x}_{2}-\mathbf{v} \tau-\mathbf{a} t \tau\right) \frac{\dot{c}(d(t, \tau))}{d(t, \tau)} w(t) d t .
$$

Now we expand as a Taylor series in $a=|\mathbf{a}|$ and make the first choices about $w(t)$. If $w(t)$ is an even function of $t$, that is $w(t)=w(-t)$, then odd powers of $t$ in the integral disappear and

$$
E[\dot{e}(\tau)]=2 \int \frac{\left[\mathbf{v} \cdot\left(\mathbf{x}_{1}-\mathbf{x}_{2}-\mathbf{v} \tau\right) \dot{c}\left(\left|\mathbf{x}_{1}-\mathbf{x}_{2}-\mathbf{v} \tau\right|\right)+O\left(a^{2}\right)\right]}{\left|\mathbf{x}_{1}-\mathbf{x}_{2}-\mathbf{v} \tau\right|} w(t) d t .
$$

Terms of order $a$ would remain here if an unsymmetric method in $\tau$ were used. Nothing in the integral now depends upon $t$ except for $w(t)$ so

$$
E[\dot{e}(\tau)] \approx 2\left[\mathbf{v} \cdot\left(\mathbf{x}_{1}-\mathbf{x}_{2}-\mathbf{v} \tau\right) \frac{\dot{c}\left(\left|\mathbf{x}_{1}-\mathbf{x}_{2}-\mathbf{v} \tau\right|\right)}{\left|\mathbf{x}_{1}-\mathbf{x}_{2}-\mathbf{v} \tau\right|} \int w(t) d t .\right.
$$

At the minimum of $e(\tau), \dot{e}(\tau)=0$, so we need to look for solutions of Equation 28 equal to zero. Suppose that the sensors are a distance $L$ apart along the $x$-axis then Equation 28 becomes 


$$
E[\dot{e}(\tau)] \propto\left[\tau\left(v_{x}^{2}+v_{y}^{2}\right)-L v_{x}\right] \frac{\dot{c}\left(\sqrt{\left(L-v_{x} \tau\right)^{2}+v_{y}^{2} \tau^{2}}\right)}{\sqrt{\left(L-v_{x} \tau\right)^{2}+v_{y}^{2} \tau^{2}}}
$$

If $\dot{c}\left(\sqrt{\left(L-v_{x} \tau\right)^{2}+v_{y}^{2} \tau^{2}}\right) \approx 0$ for all $\tau$ in Equation 28 this means that there are no correlations between the sensors. This is the case discussed in Subsection 3.2. This occurs if the minimum value of $d(0, \tau)=L v_{y} / \sqrt{v_{x}^{2}+v_{y}^{2}}$ is so large that $c(d(0, \tau)) \approx 0$. When there are no appreciable correlations between the sensors it is of course impossible to calculate the velocity by any method. This failure can be minimised by reducing $L$, ensuring that the sensors are aligned with the flow so that $v_{y}$ is as small as possible, or by increasing the correlation length by using sensors that average over a large spatial area.

If $\dot{c} \neq 0$ then Equation 28 is zero when

$$
\tau=\frac{\mathbf{v} \cdot\left(\mathbf{x}_{\mathbf{1}}-\mathbf{x}_{\mathbf{2}}\right)}{|\mathbf{v}|^{2}}+O\left(a^{2}\right)=\frac{v_{x} L}{v_{x}^{2}+v_{y}^{2}} .
$$

This means that, if we estimate the $x$-axis velocity by $\hat{v}_{x}=L / \tau$, we in fact calculate

$$
\hat{v}_{x}=\frac{L}{\tau}=L \frac{v_{x}^{2}+v_{y}^{2}}{v_{x} L}=v_{x}+\frac{v_{y}^{2}}{v_{x}} .
$$

Thus direct maximisation of the correlation between two sensors does not measure the velocity in the direction of the sensors but this more complicated function of velocities. If $v_{y}$ is small this will be a good approximation, but in other cases the results can be severely biased; the estimated velocity will be larger than the true velocity by $v_{y}^{2} / v_{x}$. The problem is discussed in Section 2 and illustrated in the left two parts of Figure 4. Even though the horizontal speeds are different in these two figures the output signals are identical. This is the major problem with one-dimensional arrays of sensors. If there is some way of calculating the vertical velocity $v_{y}$ then the bias can be eliminated by solving for $v_{x}$ as a function of $\hat{v}_{x}$ and $v_{y}$ in equation Equation 31. The obvious way to do this is to build a twodimensional sensor array with closely spaced vertical sensors so that $v_{y}$ can be separately estimated. A simple sensor that can do this is discussed later. If it is not feasible to install a new sensor another approach is to alter the vertical length scale by averaging vertically. If the sensors average over a large vertical region so that the vertical dimensions are $\lambda L$, such as in Figure 9, then the effective vertical velocity is reduced by a factor $\lambda$ so that Equation 31 becomes

$$
\hat{v}_{x}=v_{x}+\frac{v_{y}^{2}}{v_{x} \lambda^{2}}
$$




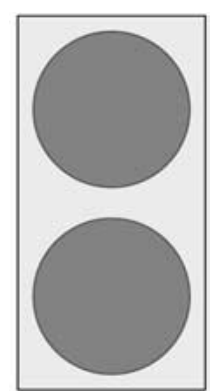

Figure 9. One-dimensional array of two sensors designed to reduce bias from vertical velocities. The active region of the sensors averages over a large vertical region to reduce bias.

Thus if the aspect ratio is 4 the error will be reduced by a factor of 16. Building a sensor with such an aspect ratio could be achieved by using a lens, appropriate choice of sensor and orientation, or by stacking sensors and combining their outputs.

\subsection{GAUSSIAN CORRELATION}

In this section we assume that the correlation function $c(x)$ is Gaussian and we also use a Gaussian weight function $w(t)$. This choice is made because the calculations are then easy to perform, and it is sufficient to demonstrate the dependence of the errors on the design parameters and the properties of the flow. Analysis could also be performed with correlation functions for hard disc systems or using correlation functions measured from the data. We do not expect this would significantly change the conclusions.

We proceed as in the previous section to analyse the method using the expectation of the derivative of $e(\tau)$. We define $d_{0}(t)=d\left(t+\tau_{0}, t-\tau_{0}\right)$, where $\tau_{0}$ is the lag that minimises the squared discrepancy. Thus

$$
d_{0}=\frac{L v_{y}}{v},
$$

which has a simple interpretation. $d_{0}$ is the point of closest approach to sensor 2 of a point moving with the flow that passes over sensor 1 at around time 0. Expanding in powers of $a$ and removing terms odd in $t$

$$
\begin{aligned}
& E\left[e\left(\tau_{0}\right)\right]=2 W_{0}\left[c(0)-c\left(d_{0}\right)\right]+O\left(a^{2}\right), \\
& E\left[\dot{e}\left(\tau_{0}\right)\right]=O\left(a^{2}\right), \\
& E\left[\ddot{e}\left(\tau_{0}\right)\right]=-2 W_{0} v^{2} \frac{\dot{c}\left(d_{0}\right)}{d_{0}}+O\left(a^{2}\right),
\end{aligned}
$$

where $W_{0}=\int w(t) d t$. More complicated expressions can be calculated for the variances, $V\left[\dot{e}\left(\tau_{0}\right)\right]$ and $V\left[\ddot{e}\left(\tau_{0}\right)\right]$, but these will not be included here. Instead we 
illustrate how these results can be used by taking $w(t)$ to be a Gaussian function of time scale $T$, and assuming that the correlation function $c$ is also a Gaussian with length scale given by $L_{c}$.

$$
w(t)=\frac{e^{-\left(t^{2} / 2 T^{2}\right)}}{2 T} \quad c(t)=C_{0} \frac{e^{-\left(t^{2} / 2 L_{c}^{2}\right)}}{\sqrt{2 \pi}} .
$$

This choice is made because the integrals for the expectations $E[e], E[\dot{e}]$ and $E[\ddot{e}]$ can all be performed exactly and the integrals for the variances $V[e], V[\dot{e}]$ and $V[\ddot{e}]$ can be easily approximated by expanding in powers of $a$. What this approximation corresponds to is assuming that $T a / v$ is small, where $v=|\mathbf{v}|$. This is a natural assumption since methods of this type will not work well if the velocity varies significantly over the window. In the following analysis we generally work to the lowest non vanishing term. There is no significant difference between a direct correlation method and a residual method.

The probability that the algorithm fails to find a minimum near the correct minimum is given by

$$
p(\text { fail }) \approx \frac{3}{4} \frac{1+\exp \left(\frac{L^{2} v_{y}^{2}}{L_{c}^{2} v^{2}}\right)}{\sqrt{1+4 T^{2} v^{2} / L_{c}^{2}}} .
$$

The numerator contains a positive exponential term with argument $L^{2} v_{y}^{2} / L_{c}^{2} v^{2}=$ $d_{0}^{2} / L_{c}^{2}$. In order for the failure probability to be small this must be of order 1 . Since the exponential term is added to 1 there is little advantage in making it very small. The term will be of order one in two situations. If the spacing of the sensors is sufficiently close that no spatial aliasing occurs for all $v_{x}$ and $v_{y}$ (Section 3.2), that occurs for $L \leq L_{c}$. Or when the angle of the velocity to the sensor axis is sufficiently small, then $v_{y} / v \leq L_{c} / L$. For most sensors built so far the spacing is at least $5 \mathrm{~mm}$, which is well above the smallest correlation lengths which will be of the order of the particle diameters, around $1 \mathrm{~mm}$. Thus there will be no detectable correlations except for velocities with angle less than $\approx \tan ^{-1}(1 / 5)$. However, the large scale features of a snow avalanche may be many metres in size (Nishimura et al. (1987)) and these will be detected as a correlation between both sensors for any velocity direction.

This result is exactly what would be expected. Equation 36 contains a factor $\dot{c}\left(d_{0}\right)$ and it is the reciprocal of this squared that appears as the numerator in Equation 38 (since $d_{0}^{2} / \dot{c}\left(d_{0}\right)^{2} \propto \exp \left(d_{0}^{2} / L_{c}^{2}\right)$ ). For many correlation functions $\dot{c}(d)=0$ for all $d$ greater than some critical value; in that case Equation 38 diverges and failure becomes a certainty.

Given that the numerator of Equation 38 is of order 1 the probability of failure can only be small if the denominator is large, which occurs if $T v / L_{c}$ is large. This also has a natural interpretation. $T v$ is the length of material that has passed over 
the sensor. Dividing this by the correlation length $L_{c}$ give the approximate number of independent samples. And this is the usual reduction in variance with sample size. If this is large and the exponential is limited, then Equation 38 becomes

$$
p(\text { fail }) \approx \frac{3}{4} \frac{L_{c}}{T v} .
$$

We focus on the case where the vertical velocities are small and consider $u_{y}=$ $O\left(a^{1 / 2}\right)$. The probability of failure, from Equation 38, will then be small if $T=$ $O\left(a^{-1 / 2}\right)$. This is the most interesting gearing because the leading order terms contain vertical velocity terms and acceleration terms. Using the results in the Appendix and Gaussian correlation and weighting functions we obtain:

$$
\begin{aligned}
& p(\text { fail }) \approx \frac{3}{4} \frac{L_{c}}{u T}+\frac{3}{16} \frac{\left[L^{2}\left(7 a_{x}^{2}+3 a_{y}^{2}\right)-L_{c}^{2}\left(2 a_{x}^{2}+5 a_{y}^{2}\right)\right] T}{u_{x}^{3} L_{c}} \\
&+\frac{3}{8} \frac{u_{y}^{2}}{u_{x}^{2}} \frac{L^{2}-L_{c}^{2}}{L_{c} u_{x} T}-\frac{3}{32} \frac{L_{c}}{u_{x}^{3} T^{3}}, \\
& E\left[\hat{v}_{x}\right]-v_{x}=\frac{v_{y}^{2}}{v_{x}}+\frac{T^{2} a^{2}}{v_{x}}, \\
& V\left[\hat{v}_{x}\right]=\frac{L_{c} v_{y}^{2}}{4 v_{x} T}+\frac{L_{c}\left(a_{y}^{2}+3 a_{x}^{2}\right) T}{8 v_{x}} .
\end{aligned}
$$

Now that the acceleration terms are included it is clear that there is a value of $T$ that minimises $p$ (fail), which scales like $v_{x} / a$ if $L \approx L_{c}$. For such large $T$, however, the bias would be of order $v_{x}$, so that though the method would be likely to find a minimum in the correct region, the error would be unacceptable. This illustrates part of the tradeoff between large $T$ maximising the chance of finding a minimum, but large $T$ also coupling to accelerations and increasing the errors. This is also shown in the variance Equation 42. Large $T$ decreases the first term in proportional to the number of independent samples $T v_{x} / L_{c}$, but increases the second term because of acceleration. The variance term will be minimised by a $T$ approximately $v_{y} / a$, which is order $a^{-1 / 2}$ as we assumed. If $T=v_{y} / a$ then the bias term will just be of the same order $v_{y}^{2} / v_{x}$. This choice of $T$ thus provides a compromise between vertical velocity errors and acceleration errors. The bias Equation 41 also shows that all the errors to this order increase the apparent mean velocity.

Once the velocity estimate $\hat{v}_{x}$ has been formed we can use these equations to estimate the errors. This can be done as follows. The expected residual will be

$$
E\left[e\left(\tau_{0}\right)\right]=C_{0} \frac{L^{2}\left(v_{y}^{2}+a^{2} T^{2}\right)}{2 L_{c}^{2} v_{x}^{2}},
$$


and the curvature

$$
E\left[\ddot{e}\left(\tau_{0}\right)\right]=\frac{C_{0} v_{x}^{2}}{L_{c}^{2}} .
$$

If we equate the measured residual $\hat{e}\left(\tau_{0}\right)$ and curvature $\hat{\ddot{e}}\left(\tau_{0}\right)$ to these equations we can estimate the errors. Using Equations 43 and 44

$$
\frac{E\left[e\left(\tau_{0}\right)\right]}{E\left[\ddot{e}\left(\tau_{0}\right)\right]}=\frac{L^{2}\left(u y^{2}+T^{2} a^{2}\right)}{2 u_{x}^{4}} .
$$

Thus the bias can be estimated as

$$
-\frac{2 u_{x}^{3}}{L^{2}} \frac{\hat{e}\left(\tau_{0}\right)}{\hat{\ddot{e}}\left(\tau_{0}\right)},
$$

The beauty of this is that it is not necessary to estimate $C_{0}$ or $L_{c}$ directly. Thus our revised estimate of the velocity is

$$
\hat{v}_{x}^{\prime}=\frac{L}{\tau_{0}}-\frac{2 u_{x}^{3}}{L^{2}} \frac{\hat{e}\left(\tau_{0}\right)}{\hat{\hat{e}}\left(\tau_{0}\right)}
$$

which is bias free to this order.

The variance equation Equation 42 can be similarly estimated; if we approximate $\left(a_{y}^{2}+3 a_{x}^{2}\right) \approx 2 a^{2}$ it becomes

$$
V[\hat{v}]=L_{c} \frac{v_{y}^{2}+T^{2} a^{2}}{4 v_{x} T}=\frac{L_{c} u_{x}^{3}}{2 T L^{2}} \frac{E\left[e\left(\tau_{0}\right)\right]}{E\left[\ddot{e}\left(\tau_{0}\right)\right]} .
$$

Unfortunately it is not possible to estimate the variance without also estimating the correlation length $L_{c}$, but this can be done using standard techniques. Equation 48 is the variance estimate of $\hat{v}$, not of the bias-corrected velocity $\hat{v}^{\prime}$. The variance of this will be larger since there is the variance of the correction term to consider. For this reason it is probably best to regard the bias correction as an error estimate rather than performing it directly. The one standard deviation confidence interval will then be roughly

$$
\left[\frac{L}{\tau_{0}}-\frac{2 u_{x}^{3}}{L^{2}} \frac{\hat{e}\left(\tau_{0}\right)}{\hat{\tilde{e}}\left(\tau_{0}\right)}-\frac{v_{x}}{L} \sqrt{\frac{L_{c} v_{x}}{2 T} \frac{\hat{e}\left(\tau_{0}\right)}{\hat{\tilde{e}}\left(\tau_{0}\right)}}, \quad \frac{L}{\tau_{0}}+\frac{v_{x}}{L} \sqrt{\frac{L_{c} v_{x}}{2 T} \frac{\hat{e}\left(\tau_{0}\right)}{\hat{\ddot{e}}\left(\tau_{0}\right)}}\right],
$$

A check on the assumptions could also be performed by using the calculated $\hat{v}_{x}$ to estimate the acceleration $a_{x}$.

The design criterion for correlation sensors can thus be summarised as follows, in decreasing order of importance: 


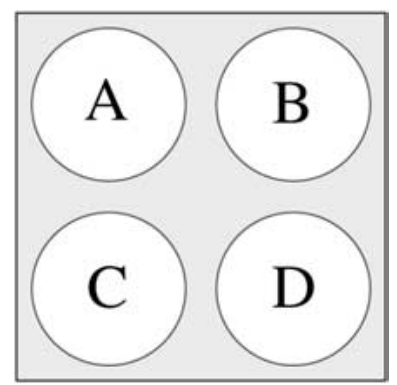

Figure 10. Two-dimensional array of four sensors designed to measure vertical and horizontal velocities. The active region of the sensors is as large as possible to reduce spatial aliasing.

$L \approx L_{c}$. Eliminates spatial aliasing and allows correlations at all velocity angles. If this is not possible then the sensor can only be operated in the range $\left(v_{y} / v\right)\left(L / L_{c}\right)=$ $O(1)$.

$f_{s}>2 v / L_{c}$. The sampling frequency must be high enough to prevent temporal aliasing.

$T v / L_{c} \ll 1$. Good sample statistics.

$T a / v^{2} \ll 1$. Low bias and variance.

$v_{y} / v \ll 1$. Low bias and variance.

Confidence intervals should be calculated according to Equation 49.

The next two sections discuss how to reduce and eliminate the errors induced by vertical velocities and accelerations.

\subsection{D FLOW}

A full discussion of design criteria for two-dimensional sensors is contained in McElwaine (2003), but a rough approach is straightforward. The most important criterion for a $2 \mathrm{D}$ sensor is that $L \approx L_{c}$ or smaller so that there are always the appropriate correlations between the sensors. Figure 10 shows a sensor with four photo-transistors arranged on a square. The optimal lag $\tau$ can be calculated between six pairs $(\mathrm{AB}, \mathrm{AC}, \mathrm{AD}, \mathrm{BC}, \mathrm{BD}, \mathrm{CD})$ in a similar way as for $1 \mathrm{D}$ sensors. Let $\tau_{x}$ and $\tau_{y}$ minimise the respective expressions

$$
\begin{aligned}
e_{x}\left(\tau_{x}\right)= & \int w(t)\left\{\left[f_{A}\left(t+\tau_{x} / 2\right)-f_{B}\left(t+\tau_{x} / 2\right)\right]^{2}+\left[f_{C}\left(t+\tau_{x} / 2\right)\right.\right. \\
& \left.\left.-f_{D}\left(t+\tau_{x} / 2\right)\right]^{2}\right\} d t, \\
e_{x}\left(\tau_{y}\right)= & \int w(t)\left\{\left[f_{A}\left(t+\tau_{y} / 2\right)-f_{C}\left(t+\tau_{y} / 2\right)\right]^{2}+\left[f_{B}\left(t+\tau_{y} / 2\right)\right.\right. \\
& \left.\left.-f_{D}\left(t+\tau_{y} / 2\right)\right]^{2}\right\} d t,
\end{aligned}
$$

where $f_{A}, f_{B}, f_{C}$ and $f_{D}$ are the signals from each photo-transistor. By combining the signals in this way, rather than calculating separate lags for each pair, the errors can be reduced. Diagonal correlation lags $\tau_{A D}$ and $\tau_{C B}$ can also be calculated 
between $A D$ and $C B$, respectively. If we let $\tau_{x}$ and $\tau_{y}$ be the minimising correlation lags then

$$
\begin{array}{ll}
\frac{L}{\tau_{x}}=\frac{v_{x}^{2}+v_{y}^{2}}{v_{x}}, & \frac{L}{\tau_{y}}=\frac{v_{x}^{2}+v_{y}^{2}}{v_{y}}, \\
\frac{L}{\tau_{A D}}=\frac{v_{x}^{2}+v_{y}^{2}}{v_{x}+v_{y}}, & \frac{L}{\tau_{C B}}=\frac{v_{x}^{2}+v_{y}^{2}}{v_{x}-v_{y}} .
\end{array}
$$

The bias and variance of each of the $\tau$ 's can be calculated using the previous methods. Then they can then be suitably combined to produce an estimate of $v_{x}$ and $v_{y}$ with minimum error. Suppose that $\sigma_{x}, \sigma_{y}, \sigma_{A D}$ and $\sigma_{C B}$ are the expected root mean squared errors. Then the minimum expected root mean squared error estimates can be calculated by minimising

$$
\begin{aligned}
\frac{\left[\frac{L}{\tau_{x}}-\frac{v_{x}^{2}+v_{y}^{2}}{v_{x}}\right]^{2}}{\sigma_{x}^{2}}+\frac{\left[\frac{L}{\tau_{y}}-\frac{v_{x}^{2}+v_{y}^{2}}{v_{y}}\right]^{2}}{\sigma_{y}^{2}}+\frac{\left[\frac{L}{\tau_{A D}}-\frac{v_{x}^{2}+v_{y}^{2}}{v_{x}+v_{y}}\right]^{2}}{\sigma_{A D}^{2}} \\
+\frac{\left[\frac{L}{\tau_{C D}}-\frac{v_{x}^{2}+v_{y}^{2}}{v_{x}-v_{y}}\right]^{2}}{\sigma_{C B}^{2}}
\end{aligned}
$$

The expression is complicated to minimise analytically, especially as the estimated squared errors, $\sigma_{x}^{2}$, etc., will be functions of $v_{x}$ and $v_{y}$. It is, however, easy to minimise numerically. If the flow were between directions $A D$ and $A B$, then $\sigma_{y}$ and $\sigma_{C D}$ would be large and the solution would be weighted towards $\tau_{x}$ and $\tau_{A D}$. The result would roughly be

$$
v_{x}=\frac{L \tau_{x}}{\tau_{x}^{2}+\left(\tau_{A D}-\tau_{x}\right)^{2}}, \quad v_{y}=\frac{L\left(\tau_{A D}-\tau_{x}\right)}{\tau_{x}^{2}+\left(\tau_{A D}-\tau_{x}\right)^{2}} .
$$

\subsection{Continuous Velocity ESTIMATION}

The errors due to accelerations in the previous analysis are caused by assuming a fixed velocity over the window width. If instead of this we try to estimate the varying function $v(t)$ at all times, it is possible to eliminate or at least reduce this source of error. Equation 17 can be naturally generalised to

$$
e[\tau]=\int\left[f_{1}(t+\tau(t) / 2)-f_{2}(t-\tau(t) / 2)\right]^{2} d t,
$$


where $\tau(t)$ is a function of time for the whole measurement period, $e[\tau]$ is now a functional and the integration is to be performed over the whole sample. In order for this to be a well posed problem it is necessary to constrain $\tau(t)$. This is done by introducing a penalty functional term

$$
\lambda \int\left(\frac{d \tau(t)}{d t}\right)^{2} d t
$$

where $\lambda$ determines the degree of smoothing enforced on $\tau$. The Euler-Lagrange equation for this system is

$$
\begin{aligned}
\lambda \frac{d \tau(t)^{2}}{d t^{2}}= & {\left[\dot{f}_{1}(t+\tau(t) / 2)+\dot{f}_{2}(t-\tau(t) / 2)\right]\left[f_{1}(t+\tau(t) / 2)\right.} \\
& \left.-f_{2}(t-\tau(t) / 2)\right] .
\end{aligned}
$$

This equation can be efficiently solved using multi-grid techniques and the velocity calculated as $v_{x}(t)=L / \tau(t)$. A full discussion will be included in future work.

\section{Results}

Experiments were carried out at the SLF chute in Davos. Preliminary results and three different methods of analysis will be briefly mentioned. A full analysis will appear in a later paper.

The velocity sensor array consists of a $5 \times 5$ matrix of photo diode/photo transistor pairs. First we analyse the data using the traditional method of Dent et al. (1998) (Equation 10). For each row of the sensor array, the cross correlation function can be computed for 10 pairs of signals corresponding to 10 combinations of each two time shifted sensor signals related to 10 different stream-wise sensor spacings.

Assuming that the velocity of the flow does not change significantly while passing the sensor array which has a stream-wise extension of $2 \mathrm{~cm}$, the accuracy of the velocities obtained from one row of the sensor array can be estimated by statistical analysis of the velocities measured by all possible sensor pair combinations of the row. Previous experimental setups of optical correlation velocity measurements in avalanches (Nishimura et al., 1993; Dent et al., 1998) were using only one pair of signals, and therefore did not allow such an error analysis.

Figure 11 shows a plot of the mean velocities obtained from all 5 sensors of row 3 and 5 of the sensor array where the error bars indicate the standard deviation of the mean velocity, $\bar{v}$, which is an average of the velocities obtained by correlation of each of the 10 sensor pairs of a row of the sensor array. The correlation functions have been evaluated using a $0.16 \mathrm{~s}$ interval every $0.012 \mathrm{~s}$. 


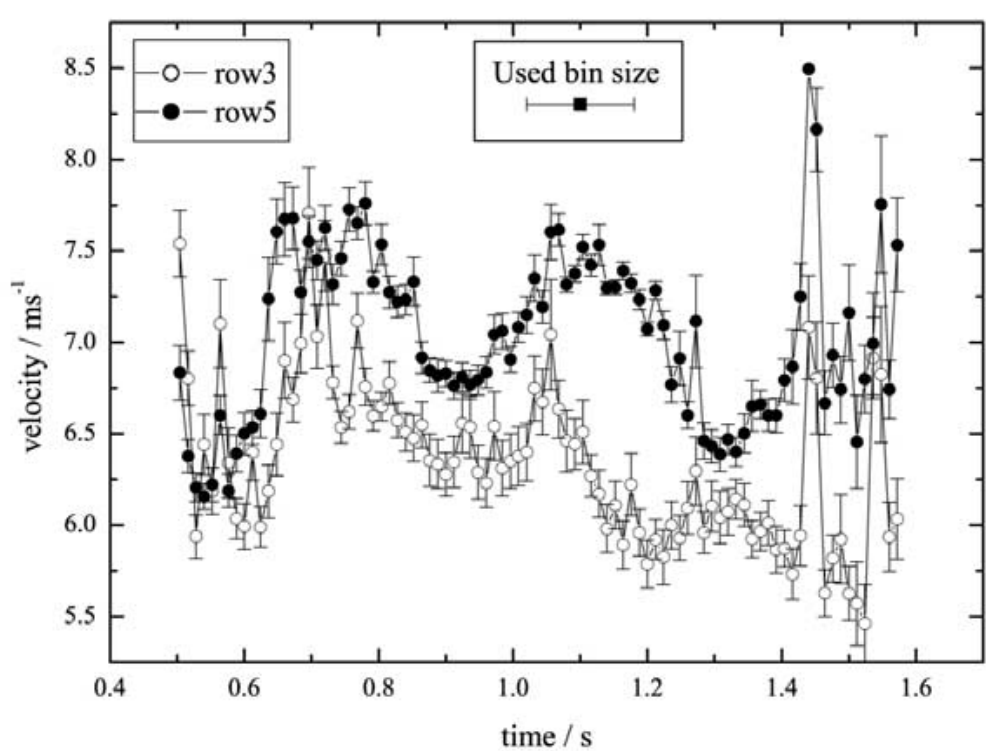

Figure 11. Velocity measurements by row 3 and 5 of the sensor array performed on April 3, 2002.

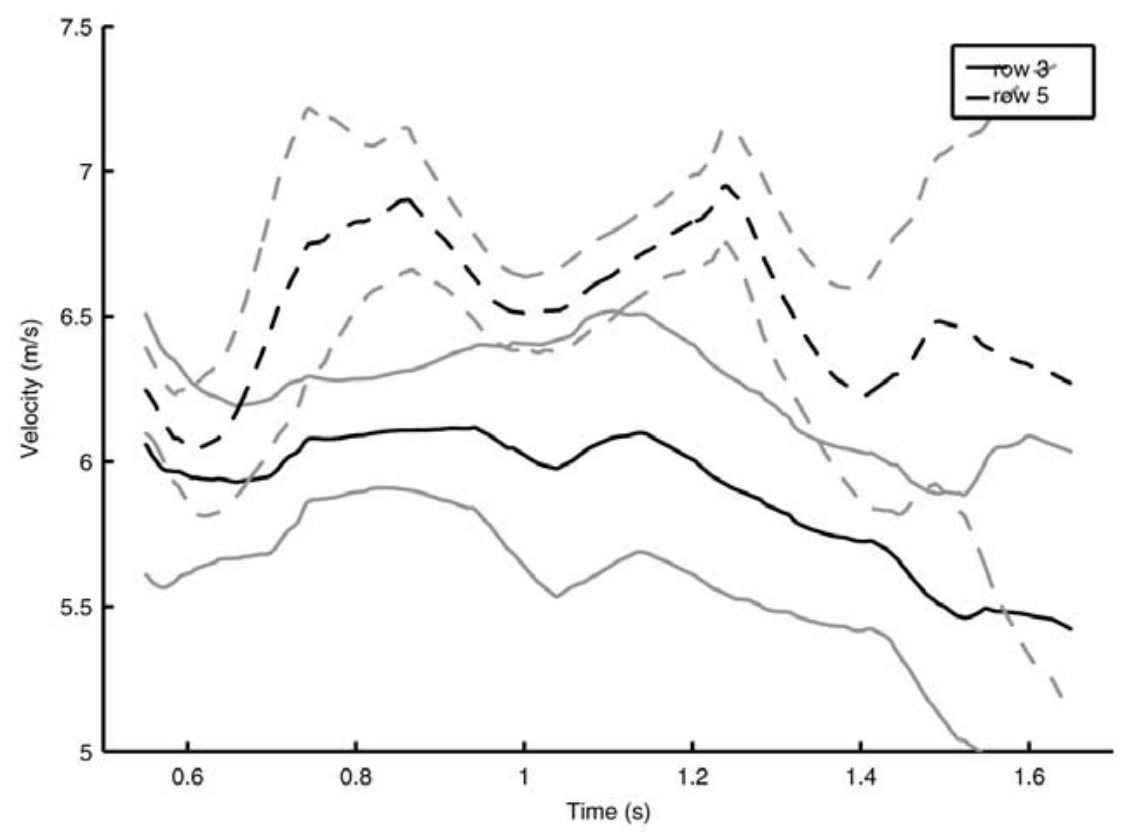

Figure 12. Continuous velocity estimates with the populations' standard deviations (grey). 
The vertical distance between row 3 and 5 of the sensor array is $2.1 \mathrm{~cm}$ where row 3 is situated $2.1 \mathrm{~cm}$ above the sliding surface. Taking into account the errors mentioned above, the velocities obtained by the two sensor rows are significantly shifted as is shown in Figure 11. Interpreting the plot with respect to the vertical spacing of the rows, the shear can be estimated to be approximately $25 \mathrm{~s}^{-1}$ in the flow layer between $2.1 \mathrm{~cm}$ and $4.2 \mathrm{~cm}$ above the sliding surface.

Figure 12 shows the velocities calculated using the continuous approach of Section 4.6. The standard deviations are calculated from the ten different velocity measurements pairs at each row height. The gross features of the two results are clearly similar but the overall velocities are considerably lower, so much so that the error intervals of the estimates do not overlap. This is not that surprising as these are not true error estimates, but instead highly correlated estimates of the variance in the velocity. They do not account for any bias effects resulting from vertical velocities, so the true horizontal velocities may be considerably lower. Secondly, the sensors are all closely positioned so that the errors between the sensors cannot be assumed to be independent. Roughly speaking, if the correlation between the errors is $\rho$, then the true standard deviation will be larger by a factor $1 /(1-\rho)$.

\section{Conclusions}

The work in this paper is still ongoing and is by no means complete. In particular a detailed analysis of the auto-correlations should be performed and also an explanation of the large differences in the results of the two analysis methods in the last section. Though correlation sensors are a very useful tool great care should be exercised in interpreting the data, and particular care has to be taken to understand the uncertainties in the estimations. In particular the usual estimate of velocity $\hat{v}_{x}=L / \tau$ is in fact an estimate of

$$
v_{x}+\frac{v_{y}^{2}}{v_{x}}+\frac{T^{2} a^{2}}{v_{x}}
$$

and will, therefore, be considerably larger then the true velocity $v_{x}$ unless $v_{y}^{2} / v_{x}^{2}$ and $T^{2} a^{2} / v_{x}^{2}$ are small. The analysis also demonstrates that it should be possible to greatly reduce the errors by using closely spaced two-dimensional arrays of sensors that are as small as the smallest correlation lengths, which are of the order of the grain size $(1 \mathrm{~mm})$.

\section{Acknowledgements}

J. N. McElwaine was supported by a University Research Fellowship from the Royal Society. The authors would like to thanks to Martin Kern for help with 
carrying out the experiments and building the sensor, and Rick Munro for many helpful comments.

\section{Appendix A. Estimating Parameters}

It is a well known result of classical statistics that the best asymptotic estimators are maximum likelihood estimators (MLE). Unfortunately to apply these methods the probability density function of the reflectivity would have to be specified by a parametric model. If this could accurately be done this would provide the best method of analysis. If a bad parametric model is applied, however, the estimators may be very poor indeed. So, until more data are available, we use a non-parametric approach which attempts to be similar to MLE methods, but without requiring a complete probabilistic description. Our methods are based on minimising a sum of residuals and, if these residuals are regarded as independent and Gaussian, then this is equivalent to an MLE estimate.

Suppose that we estimate the speed of the flow $\hat{v}_{x}=L / \hat{\tau}$ by minimising a function $e(\tau)$. To estimate the accuracy of the method we wish to find the bias or expected error $E[\hat{\tau}]$, the variance in the estimate $V[\hat{\tau}]=E\left[\hat{\tau}^{2}\right]-E[\hat{\tau}]^{2}$ and the probability that there is a minimum close to the true value. In this Appendix expressions for these values are derived.

Let $e(\tau)$ be a function of the data and assume that the true minimum occurs at $\tau_{0}$. Due to noise and other inaccuracies when we attempt to minimise $e(\tau)$, we will find the minimum at some other value $\hat{\tau} \neq \tau_{0}$. Expanding to second order

$$
e(\tau)=e\left(\tau_{0}\right)+\tau \dot{e}\left(\tau_{0}\right)+1 / 2 \tau^{2} \ddot{e}\left(\tau_{0}\right) .
$$

Thus the minimum will be found at $\hat{\tau}=-\dot{e}\left(\tau_{0}\right) / \ddot{e}\left(\tau_{0}\right)$ provided that $\ddot{e}\left(\tau_{0}\right)>0$. That is, the method will succeed if $p\left(\ddot{e}\left(\tau_{0}\right)<0\right)$ is small and the error will be $-\dot{e}\left(\tau_{0}\right) / \ddot{e}\left(\tau_{0}\right)$. The probability of failure can be bounded using Chebychev's inequality. Let $\mu=E\left[\ddot{e}\left(\tau_{0}\right)\right]>0$ and $\sigma^{2}=E\left[\ddot{e}\left(\tau_{0}\right)^{2}\right]-\mu^{2}$. Then

$$
\begin{aligned}
P\left(\ddot{e}\left(\tau_{0}\right)<0\right) & \left.=P\left(a-\ddot{e}\left(\tau_{0}\right)>a\right) \leq P\left(\mid \ddot{e} \tau_{0}\right)-a \mid>a\right) \\
& \leq \frac{E\left[\left|\ddot{e}\left(\tau_{0}\right)-a\right|^{2}\right]}{a^{2}}=\frac{\sigma^{2}+(\mu-a)^{2}}{a^{2}} .
\end{aligned}
$$

This upper bound is true for all $a$, and is minimised by $a=\left(\sigma^{2}+\mu^{2}\right) / \mu$, so

$$
P\left(\ddot{e}\left(\tau_{0}\right)<0\right) \leq \frac{\sigma^{2}}{\sigma^{2}+\mu^{2}} \approx \frac{\sigma^{2}}{\mu^{2}} .
$$

This shows that the method will have a high probability of success if $V\left[\ddot{e}\left(\tau_{0}\right)\right] /$ $E\left[\ddot{e}\left(\tau_{0}\right)\right]^{2}$ is small. This bound is conservative and in many cases $\ddot{e}\left(\tau_{0}\right)$ will be 
well approximated by a normal distribution since it is the sum of many random variables. In this case the probability of failure will be estimated as $P(W \leq 0)=$ $1-\operatorname{erf}\left(\frac{\mu_{W}}{\sigma_{W}}\right)$, where $\operatorname{erf}(x)$ is the error function. The bias of the method is $E[\hat{\tau}]=$ $E\left[\frac{\dot{e}\left(\tau_{0}\right)}{\ddot{e}\left(\tau_{0}\right)}\right]$. Approximating $\dot{e}\left(\tau_{0}\right)$ and $\ddot{e}\left(\tau_{0}\right)$ as independent gives $E[\hat{\tau}]=$ $E\left[\dot{e}\left(\tau_{0}\right)\right] E\left[1 / \ddot{e}\left(\tau_{0}\right)\right]$. The method only finds a local minimum when $\ddot{e}>0$ and, since this occurs with high probability only when $\sigma^{2} / \mu^{2}$ is small, we can write $E\left[1 / \ddot{e}\left(\tau_{0}\right)\right] \approx 1 / E\left[\ddot{e}\left(\tau_{0}\right)\right]$ so that $E[\hat{\tau}]=E\left[\dot{e}\left(\tau_{0}\right)\right] / E\left[\ddot{e}\left(\tau_{0}\right)\right]$. The variance of the estimate $V[\hat{\tau}]$ can be calculated using the same approximations as before:

$$
V[\hat{\tau}]=\frac{V\left[\dot{e}\left(\tau_{0}\right)\right]}{E\left[\ddot{e}\left(\tau_{0}\right)\right]^{2}}=\frac{V\left[\dot{e}\left(\tau_{0}\right)\right]}{E\left[\ddot{e}\left(\tau_{0}\right)\right]^{2}} .
$$

Since the velocity is estimated using the rule $\hat{v}_{x}=L / \hat{\tau}$, the mean error in the estimated velocity is

$$
\begin{aligned}
E\left[\hat{v}_{x}-v_{x}\right] & \approx \frac{L}{E[\hat{\tau}]}-\frac{L}{v_{x}} \approx \frac{L}{\tau_{0}-E\left[\dot{e}\left(\tau_{0}\right)\right] / E\left[\ddot{e}\left(\tau_{0}\right)\right]}-\frac{L}{\tau_{0}} \\
& \approx \frac{L}{\tau_{0}^{2}} \frac{E\left[\dot{e}\left(\tau_{0}\right)\right]}{E\left[\ddot{e}\left(\tau_{0}\right)\right]}=\frac{v_{x}^{2}}{L} \frac{E\left[\dot{e}\left(\tau_{0}\right)\right]}{E\left[\ddot{e}\left(\tau_{0}\right)\right]} .
\end{aligned}
$$

This assumes that the error is small compared with the true velocity. The variance in the estimated velocity can be estimated similarly

$$
V\left[\hat{v}_{x}\right] \approx \frac{v_{x}^{4}}{L^{2}} \frac{E\left[\dot{e}\left(\tau_{0}\right)^{2}\right]}{E\left[\ddot{e}\left(\tau_{0}\right)\right]^{2}}
$$

These two equations 62 and 63 in conjunction with the following equation which gives the probability that the method fails

$$
P\left(\ddot{e}\left(\tau_{0}\right)<0\right)=\frac{V\left[\ddot{e}\left(\tau_{0}\right)\right]}{E\left[\ddot{e}\left(\tau_{0}\right)\right]^{2}}
$$

give a means quantitatively to evaluate different sensor designs and analysis algorithms. They can also be used to estimate the degree of uncertainty in a measurement, that is to calculate confidence limit or error intervals. To do this we need a means of estimating the various expectations in terms of the observed data. $E\left[\ddot{e}\left(\tau_{0}\right)\right]$ can be approximated as $\ddot{e}(\hat{\tau})$ in the same way as maximum likelihood estimation. This cannot be done, however, for $E\left[\dot{e}\left(\tau_{0}\right)\right]$ since at the minimum, of course, $\dot{e}(\hat{\tau})=0$. There is no general method for approximating this or the variances $V\left[\dot{e}\left(\tau_{0}\right)\right]$ and $V\left[\ddot{e}\left(\tau_{0}\right)\right]$, but given a model for the underlying data these can be approximated in terms of the total residual $e(\hat{\tau})$. 


\section{References}

Dent, J. D., Burrel, K. J., Schmidt, D. S., Louge, M. Y., Adams, E. E., and Jazbutis, T. G.: 1998, 'Density, Velocity and Friction Measurements in a Dry-snow Avalanche', Ann. Glaciol. 26, 247252.

Jähne, B.: 1997, Digital Image Processing. Concepts, Algorithms, and Scientific Applications, 4th ed., Springer, Berlin.

McElwaine, J. N.: 2003, 'Calculation of Two-Dimensional Avalanche Velocities From OptoElectronic Sensors', to appear Ann. Glac.

Nishimura, K., Maeno, N., and Kawada, K.: 1987, 'Internal Structures of Large-Scale Avalanches Revealed by a Frequency Analysis of Impact Forces', Low. Temp. Sci., Ser. A 46, 91-98. In Japanese with English Summary.

Nishimura, K., Maeno, N., Sandersen, F., Kristensen, K., Norem, H., and Lied, K.: 1993, 'Observations of the Dynamic Structure of Snow Avalanches', Ann. Glaciol. 18, 313-316.

Tiefenbacher, F. and Kern, M. A.: 2003, 'Experimental Devices to Determine Snow Avalanche Basal Friction and Velocity Profiles', Cold Reg. Sci. Tech. to appear. 